\section{Pulmonary eosinophilia due to Trichomonas}

\section{tenax}

\author{
Ali El Kamel, Naceur Rouetbi, \\ Mohamed Chakroun, Mohamed Battikh
}

\begin{abstract}
A 39 year old woman was admitted with fever and an exacerbation of asthma. The chest radiograph showed extensive bilateral lung infiltrates, but transtracheal needle aspiration did not reveal any infectious organisms. The bronchoalveolar fluid lavage contained a large number of Trichomonas tenax and an increased percentage of eosinophils. Corticosteroids provided a dramatic response. Because of the relatively high frequency of colonisation of the human mouth by $T$ tenax (about $20 \%$ ), this organism should be considered in all cases of pulmonary eosinophilia.
\end{abstract}

(Thorax 1996;51:554-555)

Keywords: Trichomonas tenax, asthma, pulmonary eosinophilia.

Pleuropulmonary manifestations of Trichomonas tenax infections were first described in $1867^{1}$ but have been reported only rarely since then. Most of the reports concern invasion of the respiratory tract. We describe a pulmonary hypersensitivity reaction to $T$ tenax infection in a patient with asthma.

\section{Case report}

A 39 year old woman with asthma was referred to our department for evaluation of persistent exacerbations of her asthma symptoms and fever. Her asthma was known to be non-allergic (IgE $80 \mathrm{IU} / 1$, skin prick tests to common allergens were negative). Physical examination revealed central cyanosis, crackles and wheeze. The chest radiograph (fig 1) demonstrated extensive bilateral pulmonary infiltrates. Laboratory data showed a peripheral blood eosinophilia $(6960 / \mathrm{ml})$ and an erythrocyte sedimentation rate of $60 \mathrm{~mm}$ in the first hour. Pulmonary function tests gave the following results: forced vital capacity $(\mathrm{FVC})=1 \cdot 391$ (42\% predicted), forced expiratory volume in one second $\left(\mathrm{FEV}_{1}\right)=0.961$ (37\% predicted), $\mathrm{FEV}_{1} / \mathrm{FVC}=69 \%$ predicted, single lung transfer factor $\left(T_{L C O}\right)=53 \%$ predicted, transfer coefficient $(\mathrm{KCO})=60 \%$ predicted; gas exchange was impaired $\left(\mathrm{PaO}_{2}=7 \cdot 4 \mathrm{kPa}\right)$.

A pulmonary infection was considered likely and a transtracheal needle aspiration was performed but revealed no infectious organisms. Empirical broad spectrum antibiotic therapy failed to improve the persistent fever and appearance of the chest radiograph; the pulmonary infiltrates persisted and were not migratory. There were no fungi in expectorated sputum samples. Precipitins and skin prick test for Aspergillus fumigatus were negative, and the RAST test (Aspergillus specific IgE) was also negative. Parasitic causes were investigated by serological studies for common parasites. Urine and stool microscopic examination and cultures were all negative. No drugs were taken by the patient except her regular treatment for asthma (inhaled corticosteroids, inhaled salbutamol and oral théophylline). There was no evidence of systemic disease at the time of diagnosis nor during the subsequent 34 months of follow up. There were no signs of any vasculitis or connective tissue disorder and there were no skin or joint lesions. Electrocardiography and echocardiography gave normal results. Immunological tests for LE cells, antinuclear and anti-DNA antibody were negative and the C-reactive protein levels were normal at $8 \mathrm{mg} / 1$. Bronchoalveolar lavage was performed and showed a marked increase in the percentage of eosinophils $(43 \%)$ together with a large number of $T$ tenax. Repeated direct examinations and culture of mouth washings revealed the presence of oral $T$ tenax. The diagnosis of pulmonary eosinophilia due to $T$ tenax was suspected.

Hydrocortisone in a dose of $300 \mathrm{mg}$ daily was started and the symptoms improved rapidly with the infiltrates resolving completely on the chest radiograph by the eighth day (fig 2 ). The eosinophil count had dropped to $255 / \mathrm{ml}$ by the 14th day.

Pulmonary function tests performed 15 days after starting treatment with hydrocortisone were nearly normal: $F V C=2 \cdot 81$ ( $84 \%$ predicted), $\mathrm{FEV}_{1}=2 \cdot 11$ ( $80 \%$ predicted), with a normal TLCo $(98 \%)$. Corticosteroid therapy was continued for three weeks at the same dose, then tapered down and discontinued after

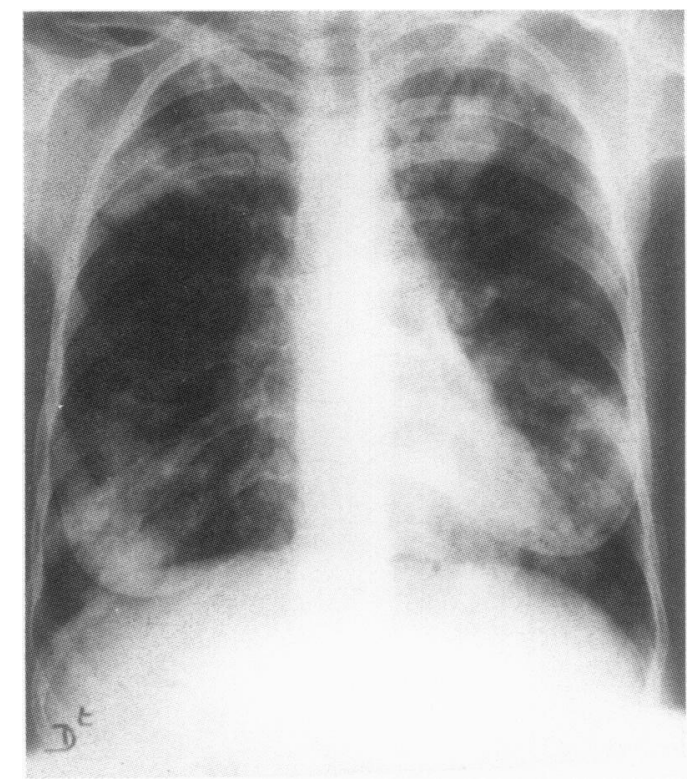

Figure 1 Chest radiograph at admission showing extensive bilateral pulmonary infiltrates. 


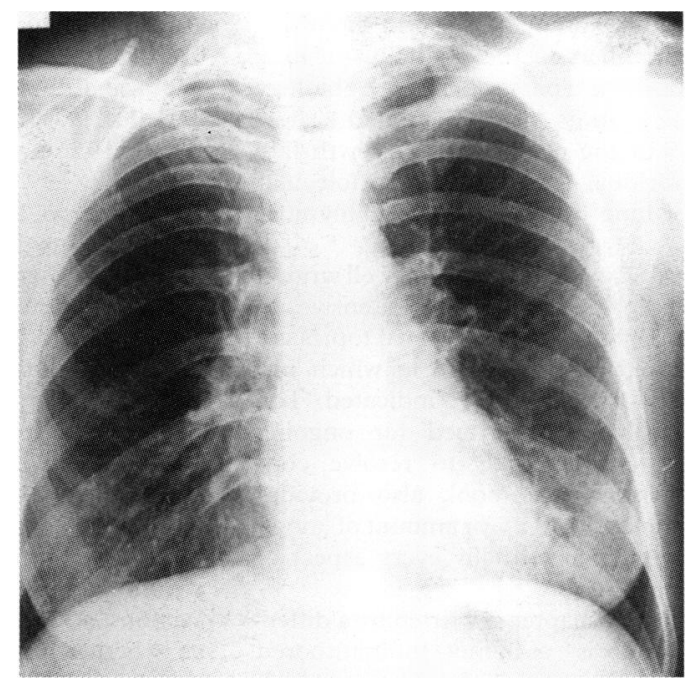

Figure 2 Chest radiograph on the eighth day of treatment with hydrocortisone.

two months. As the dose of hydrocortisone was reduced, metronidazole was added for 10 days to avoid relapse. There was no relapse on follow up, now at 34 months. The asthma is well controlled with inhaled steroids ( $1000 \mu \mathrm{g}$ daily) and inhaled salbutamol when needed.

\section{Discussion}

Trichomonas tenax is one of three species of Trichomonas found in man. It is a piriform flagellate known only in its trophozoid stage. It measures $6 \cdot 5-7 \cdot 5 \mu \mathrm{m}$ and has four free flagellae of equal length and a fifth one on the margin of the undulating membrane. ${ }^{2}$ Although the method of transmission of $T$ tenax is not known, exposure results from droplet sprays from the mouth, kissing, or from contaminated dishes. It is a harmless commensal of the human mouth which lives in the tartar around teeth and in carious cavities. Its incidence in the human mouth is about $20 \%$, depending on oral hygiene and exposure. ${ }^{3}$ The invasion of the human respiratory tract by Trichomonas was first described in 1867 but it has beeen found mainly in necrotic tissue associated with chronic and debilitating diseases such as lung abscess. ${ }^{4}$ However, trichomonads have also been reported to exist in non-necrotic lung parenchyma. ${ }^{5}$ The organism can be easily seen by direct microscopic examination of fresh unfixed material, as in our case. Detection in fixed and stained slides is more difficult than direct examination of fresh tissue.

In our case we suggest $T$ tenax caused pulmonary eosinophilia by hypersensitivity reactions. To our knowledge this type of manifestation due to $T$ tenax has not been reported. The differential diagnosis of diffuse infiltrates on chest radiographs is wide and includes bronchiolitis obliterans organising pneumonia (BOOP), sarcoidosis, vasculitis, idiopathic pulmonary eosinophilic syndrome, and other secondary pulmonary eosinophilias resulting from specific reactions to either drugs or other parasitic infestations.

We believe that $T$ tenax should be included in the differential diagnosis of pulmonary eosinophilia, particularly if no other aetiology is found.

1 Leyden E, Jaffe M. Ueber putride (fotide) sputa nebst einigen Bemerkungen ueber Lungenbrand und putride Bronchitis. Bemerkungen ueber Lungenbrand und put
Deutsch Arch Klin Med 1867;2:488-519.

2 Memik F. Trichomonads in pleural effusion. fAMA 1968; 204:211-2.

3 Rousset JJ, Larouze B. Protozooses du tube digestif. Encycl Med Chir Paris, Maladies Infectieuses 1981;8084.

4 Walton BC, Bacharach T. Occurrence of Trichomonas in the respiratory tract. Report of three cases. $\mathcal{F}$ Parasitol 1963; 49:35-8. 5 Parisot J, Simonin F. Gangrene pulmonaire et trichomonas.
CR Soc Biol 1921;85:1077-9. 